Forum 2020 · 35:1

https://doi.org/10.1007/s12312-019-00744-4

(c) Springer Medizin Verlag GmbH, ein Teil von Springer Nature 2020

\section{(9}

Check for
updates

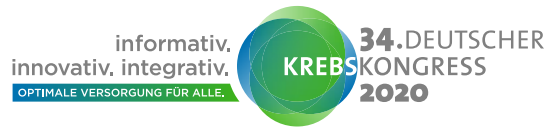

Liebe Leserinnen und Leser,

liebe Kolleginnen und Kollegen,

der größte onkologische Fachkongress im deutschsprachigen Raum - der Deutsche Krebskongress (DKK) - findet vom 19. bis 22. Februar 2020 im City Cube Berlin statt.

Unter dem Motto „informativ. innovativ. integrativ. Optimale Versorgung für alle." diskutieren Vertreter ${ }^{\star}$ innen aus Wissenschaft, Medizin, Gesundheitswesen, Politik und Pflege in rund 300 Sitzungen über neueste Erkenntnisse aus der Forschung und Versorgung von Krebspatient*innen. Auch für den wissenschaftlichen Nachwuchs wird es speziell zugeschnittene Informationsangebote geben.

Zu den Schwerpunkten auf dem Kongress zählt die Vorstellung innovativer Therapien - wir wollen diskutieren, wie sie sich in die bestehende Versorgung einfügen, so dass die Betroffenen optimal davon profitieren. Die Komplexität der Erkrankung erfordert eine enge Zusammenarbeit verschiedenster Fachrichtungen. Deshalb ist es mir wichtig, alle an der Versorgung von Krebspatientinnen und -patienten beteiligten Berufsgruppen wie etwa die Chirurgie, Strahlentherapie, medikamentöse Tumortherapie, Pa-

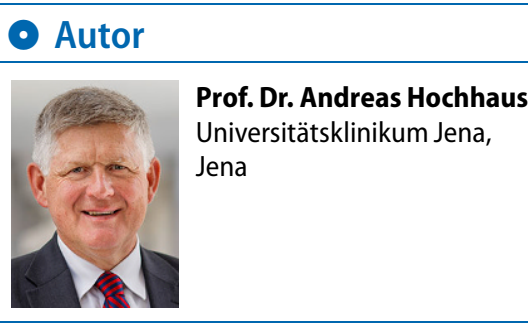

\title{
Andreas Hochhaus
}

Abt. Hämatologie und Internistische Onkologie, Klinik für Innere Medizin II, Universitätsklinikum Jena, Jena, Deutschland

\section{Editorial}

thologie, Radiologie und Pflege - zu integrieren.

Mit einem Studierendentag und Sitzungen für junge Onkolog*innen richtet sich der DKK auch gezielt an den wissenschaftlichen und ärztlichen Nachwuchs. Jedes Jahr erkranken in Deutschland 500.000 Menschen neu an Krebs. Zeitgleich gibt es einen enormen Wissenszuwachs in der Onkologie. Nur wenn wir den wissenschaftlichen Nachwuchs für eine Karriere auf diesem Feld begeistern und bestmöglich ausbilden, können wir mit diesen Entwicklungen Schritt halten.

Neben den speziell auf den Nachwuchs zugeschnittenen Programmpunkten wird es aufdem DKK Plenarsitzungen $\mathrm{zu}$ medizinischen und versorgungsrelevanten Inhalten geben, wie beispielsweise zur personalisierten antigenbasierten Immuntherapie, zu Therapiekonzepten in der Geriatrie und zur frühen Integration von Palliativversorgung. Darüber hinaus stehen interaktive Tumorkonferenzen und Pro-und-Kontra-Debatten zu medizinischen Fragestellungen auf dem Programm. In gesundheitspolitischen Foren haben die Teilnehmer*innen außerdem die Möglichkeit, die politischen Herausforderungen der Krebsmedizin in unserem Gesundheitswesen zu diskutieren.

Diese Ausgabe des FORUM widmet sich aktuellen gesundheitspolitischen und medizinischen Themen, die Gegenstand der Diskussion auf dem DKK sein werden. Demographische Entwicklung von Patienten und Ärzten, Versorgung in strukturschwachen Regionen, Karríerewege des onkologischen Nachwuchses, Krebs bei Jugendlichen und jungen Erwachsenen, sowie innovative Entwicklungen am Beispiel der CAR-TZell-Therapie.
Ich würde mich freuen, Sie im Namen der Veranstalter des Kongresses, der Deutschen Krebsgesellschaft und der Stiftung Deutsche Krebshilfe, auf dem 34. DKK in Berlin begrüßen zu dürfen.

Mit freundlichen Grüßen

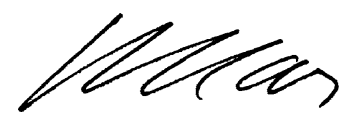

Prof. Dr. Andreas Hochhaus

Kongresspräsident

\section{Korrespondenzadresse}

Prof. Dr. Andreas Hochhaus

Abt. Hämatologie und Internistische Onkologie, Klinik für Innere Medizin II, Universitätsklinikum Jena

Am Klinikum 1, 07740 Jena, Deutschland

Andreas.Hochhaus@med.uni-jena.de

Interessenkonflikt. A. Hochhaus gibt an, dass kein Interessenkonflikt besteht. 